\title{
THEORY OF DISSIPATIVE DRIFT INSTABILITIES IN SHEARED MAGNETIC FIELDS
}

LIU CHEN, P, N, GUZDAR' J. Y, HSU, $P$. K. KAW, $C$, OBERMAN, AND R. WHITE

\section{PLASMA PHYSICS LABORATORY}

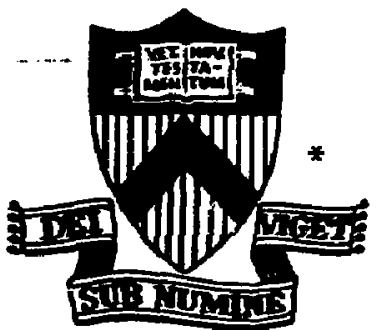

\section{PRINCETON UNIVERSITY PRINCETON, NEW JERSEY}

Th1s work was supported by the U. S. Department of Energy Contract No. EY-76-C-02-3073. Reproduction, translation, publication, use and disposal, in whole or in part, by or for the United States Government is permitted. 


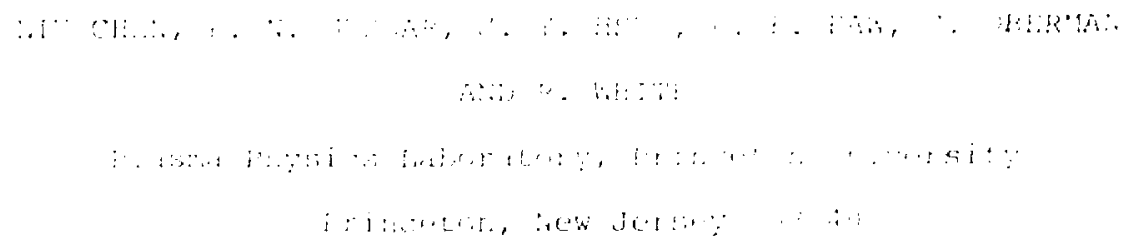

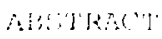

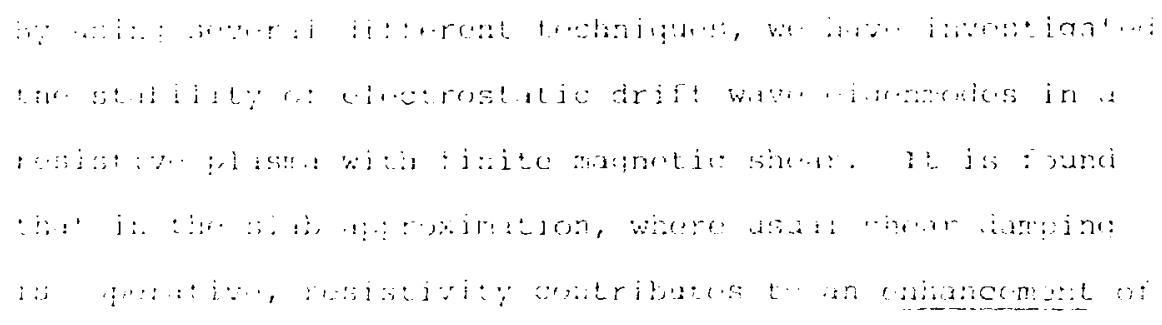

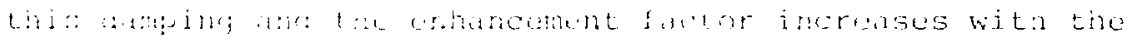

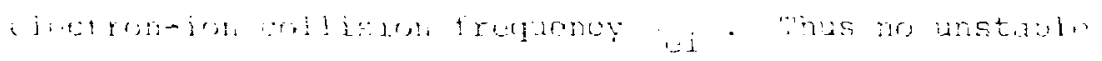

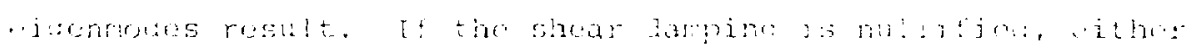

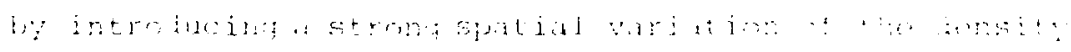

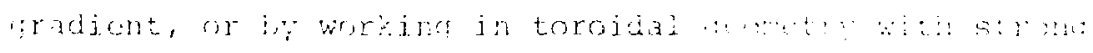

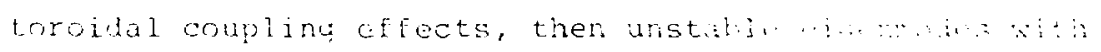

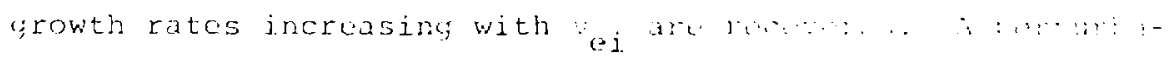

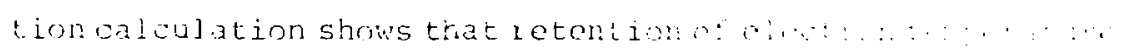

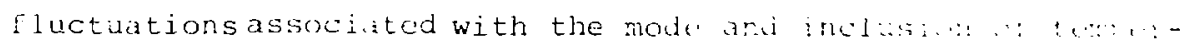
ature graidents does not alter these conolusjons. $\quad \therefore$ : $\because: \because \because:$

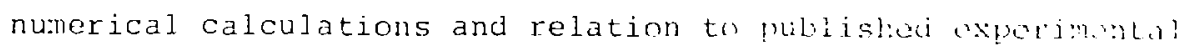
results are also presented.

Present Address: Gulf General Atomic, san Diego, $\mathrm{CA}$ 


\section{INTTRODUCTION}

It is generally recognized that low-frequency drift wave fluctuations, excited in confined plasmas by the release of expansion free energy through wave-particle ("collisionless") or collisional ("dissipative") interaction, may very well be contrjbuting to anomalous plasma transport across magnetic field lines [1]. In toroidal confinement devices like tokamaks, stellarators, multipoles etc., one therefore uses magnetic shear to stabilize these waves [2]. In a theoretical description of sur:h systems, the usual local approximations break down and one instead carries out a stability analysis for the relevant eigenmodes [3]. For collisionless drift waves driven by density gradients, such an eigenmode analysis was first carricd out in the slab approximation by Pearlstein and Berk [4]. Using a perturbation technique, they purported to show that unstable drift wave eigenmodes may result, provided the shear is weaker than a critical value. These modes are peaked around the moderational surface (defined by $\mathrm{r}_{\sim} \cdot \mathrm{B}=0$ ) and have outward propacating energy far away. To our knowledge, such an analysis for dissipative drift waves has not been carried out in any detail, although such waves may be playing an important role in certair regions of parameter and configuration space, even for high-temperature plasmas such as in tokamaks. The only previous works somewhat related to this topic that we have been able to find in the literature are a study of convective amplification of resistive drift waves without inclusion of the ion-sound term [5], and some crude attempts at estimating 
the shear stabilization criterion for these waves $[3,6]$.

In this paper we have examined the stability of resistive drift wave eigenmodes in the electrostatic approximation. Using a variety of methods, we come to the surprising conclusion that in a slab plasma with finite magnetic shear, drift wave eigenmodes are never unstable. The inclusion of resistivity is shown to further enhance the shear damping of drift wave eigenmodes by a factor which increases with the electron-ion collision frequency $v_{e i}$. We find further that if the shear damping effects are nullified, either by localizing the mode energy with a strong spatial variation of the density gradient $[7,8]$ or by using strong toroidal coupling effects [9], then unstable eigenmodes are recovered and the growth rates increase with $v_{e i}$. Very recent work with collisionless drift waves [10-12] has shown that similar conclusions hold there and that. the earlier results of Pearlstein and Berk and others $[4,13]$ were in error because of the use of some questionable approximations. The general conclusion then, is that shear damping is rather effective in stabilizing drift wave eigenmodes and that the so called universal instability is actually nonexistent. It should be emphasised, however, that even when there are no unstable eigenmodes, the possibility cf a substantial convective amplification of propagating wave packets is not ruled out $[14,15]$.

This paper is organized as follows: In Sec. 2, the physical problem is set up and the basic equations are derived. The eigenmode problem is solved by a matched asymptotic treatment in Sec. 3. In Sec. 4 we describe a WKB analysis of the problem. 
This is valid in certain regions of parameter space and entirely agrees with the matched asymptotic treatment in the appropriate limit. Section 5 gives the details of a first order perturbation theory which again recovers a limiting case of the asymptotic treatment of sec. 3. In this section, we also extend the perturbation theory to include (a) the effect of finite electron thermal conductivity along the magnetic field, and (b) any ambient normal or reverse electron temperature gradients. We conclude that the stability results obtained previously, remain essentially unchanged. Finally, since most of the above techniques are valid only in restricted regions of parameter space, we further corroborate and extend the results by a direct numerical integration of the differential equation describing the eigenmode. These results are presented in Sec. 6 .

\section{BASIC EQUATIONS}

Consider first a plasma of plane slab geometry with both, tho density variation and magnetic shear in $\mathbf{x}$ i.e., $N \equiv N(x),{\underset{\sim}{0}}_{0}(x)=B_{0}\left({\underset{\sim}{z}}_{z}+x_{\sim} e_{y} / L_{s}\right)$. Here, $I_{s}$ is the shear scale length. In this section, we shall assume the parailel electron thermal conductivity to be infinite and treat the electron dynamics as isothermal. (The effects of finite electron temperature fluctuations are treated in Sec. 5.) Ions will throughout be treated as a cold species. Using a fluid description for the dynamics of electrons as well as ions and assuming potential perturbations of the form $\phi(\underset{\sim}{x}, t)=\phi(x) \exp [\mathbf{i}$ $\left.\left(k_{y} y-u t\right)\right]$, we may write the linearized equations of 
continuity and motion as

$$
w n_{j}+i \underset{\sim}{i} \cdot\left[n(x) \underset{\sim}{v_{\perp}}\right]_{j}-k_{\| l} v_{\| j}=0
$$

and

$$
w v_{\| j}=k_{\|}\left(\frac{g}{m}\right)_{j} \phi-i v_{j j}, \quad\left(v_{11 j}-v_{11 j}{ }^{\prime}\right)+k_{\| 1}\left(\frac{T}{m N}\right)_{j}{ }^{n}
$$

$$
\begin{aligned}
& \text { Here, } j, j^{\prime}=e, i, j \neq j^{\prime}, k_{\|}=k_{y} x / L_{s} \equiv k_{\|}^{\prime} x \text {, } \\
& \underset{\sim}{\nabla_{L}}=i k_{y \sim y} e_{\sim x}+e_{x} d / d x, v_{i e}=-v_{e i} m_{e} / m_{i}, T_{i}=0,
\end{aligned}
$$

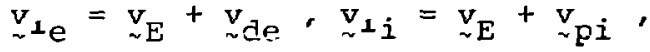

$$
\begin{aligned}
& \underset{\sim}{v_{E}}=-c\left(\underset{\sim}{\nabla} \phi \times{\underset{\sim}{B}}_{0}\right) / B_{O}^{2} \\
& \underset{\sim}{v_{d e}}=-\left(C T_{3} / \mathrm{eB}_{O}^{2} \mathrm{~N}\right)\left(\underset{\sim}{\mathrm{B}_{0}} \times \underset{\sim}{\nabla N}\right) \text {. }
\end{aligned}
$$

and

$$
\underset{\sim \mathrm{p} i}{\mathrm{v}}=-\left(\mathrm{c} \omega / \mathrm{B}_{\mathrm{o}} \omega_{\mathrm{ci}}\right) \nabla_{\perp} \phi
$$

Note that ion parallel (to $\underset{\sim 0}{B}$ ) motion is retained here so that shear damping effects due to ion-sound waves are present [4]. Note also that Eqs. (2.1)-(2.3) can be obtained using drift-kineti? equations with density conserving Krook collisional operators and working in the limit $v_{e i}>k_{\|} v_{e}, \omega$. 
Equations (2.1)-(2.3) may be used to express $n_{e}$ and $n_{i}$ in terms of $\phi$. The quasi-neutrality condition then yields the following eigenmode equation

$$
f_{s}^{2}\left(\frac{d^{2}}{d x^{2}}-k_{y}^{2}\right) \phi-\frac{x^{2}}{x^{2}-i x_{R}^{2}}\left[1-\frac{\omega_{*}{ }^{(x)}}{\omega}-\frac{x^{2}}{x_{s}^{2}}\right] \phi=0
$$

where $\mathrm{x}_{\mathrm{R}}{ }^{2}=\omega v_{\mathrm{ei}} / \mathrm{x}_{\|}{ }^{2} \mathrm{v}_{\mathrm{Te}}{ }^{2}, \mathrm{v}_{\mathrm{Te}}{ }^{2}=\mathrm{T}_{\mathrm{e}} / \mathrm{m}_{\mathrm{e}} \cdot \mathrm{x}_{\mathrm{s}}{ }^{2}=\omega^{2} / \mathrm{k}_{\|}{ }^{2} \mathrm{C}_{\mathrm{s}}{ }^{2}$, $c_{s}^{2}=T_{e} / m_{i}, \rho_{s}^{2}=c_{s}^{2} / w_{c i}{ }^{2}, w_{*}(x)=k_{y} \rho_{s} C_{s} / L_{n}(x)$ and $\left.\left[L_{n}(x)\right]^{-1}=k \ln n(x) / d x\right]$. The first term in Eq. (2.4) comes from the ion polarization drift, the coefficient $x^{2} /\left(x^{2}-i x_{R}^{2}\right)$ from the resistive parallel electron motion and the $\left(x^{2} / x_{s}{ }^{2}\right)$ term from parallel ion motion.

In considering the problem with a spatial variation of $\omega_{*}$, we treat the simple case in which $\omega_{*}$ is peaked at the mode-rational surface at $x=0$ and has a parabolic profile viz:

$$
\omega_{\star_{e}}(x) \cong w_{* 0}\left(1-\frac{x^{2}}{L_{\star}^{2}}\right)
$$

Equation (2.4) with $\omega_{*_{e}}$ constant or a function of $x$ [given by Eq. (2.5)] is the basic differential equation for the resistive drift mode in the slab approximation. For $x_{s} \rightarrow \infty, x \ll x_{R}$ and $w_{*_{e}}$ constant, Coppi, et al., [5] demonstrated that no absolutely unstable eigenmodes $\epsilon x i s t$ and only convective amplification may occur. Moiseev and Sagdeev [8] analysed the 
eigenvalue problem for Eq. (2.4) with $\mathrm{k}_{\|}=\mathrm{k}_{\|}{ }^{\prime} \mathrm{x}=$ constant (no shear), $\omega_{\star_{e}}$ given by Eq. (2.5), $x_{s}+\infty$ and $x \ll x_{R}$.

In the subsequent sections we shall address ourselves to the general eigenvalue problem posed by Eqs. (2.4) and (2.5). Since the equations are symmetric with respect to $\mathbf{x}$, the solutions obviously exhibit parities. This fixes one boundary condition, e.g., we may have even solutions with $\phi^{\prime}=0$ at $x=0$ or odd solutions with $\phi=0$ at $x=0$. The second boundary condition is for large $|x|$, where we look for outgoing waves. (This is the same as well-behaved solutions at $|x| \rightarrow \infty$ for $\operatorname{Im} \omega>0$ ). The latter condition is identical to the one proposea by Pearlstein and Berk [4] for the collisionless problem.

Before proceeding with a solution of the eigenvalue problem, we wish to derive the corresponding differential equation for resistive drift waves in an axisymmetric toroidal plasma with concentric, circular magnetic surfaces. In terms of the usual $(r, \theta, \xi)$ coordinates, we express the perturbations in the following form

$$
\phi(x, 0, \xi, t)=\exp \left[i\left(n \xi-m_{0} \theta-\omega t\right)\right] \tilde{\phi}(\theta, r),
$$

and

$$
\tilde{\phi}(\theta, r)=\sum_{\ell} \phi(\ell, s) \exp (-i \ell \theta) .
$$


Here $r, \theta$, and $\xi$ correspond, respectively, to the (minor) radial, poloidal and toroidal coordinates. $\mathrm{n}$ is the toroidal mode number. The $\theta$ dependence of $\tilde{\phi}$ arises because the toroidal magnetic field has $\theta$ dependence, viz.

$$
\quad \stackrel{\mathrm{B}}{\sim}_{0}=\mathrm{B}_{\mathrm{T} \sim \xi} \mathbf{e}_{\xi}+\mathrm{B}_{\theta_{\sim}}^{e_{\theta}}
$$

and

$$
B_{0} \simeq \bar{B}_{0}\left(1-\frac{r}{\bar{R}} \cos \theta\right) .
$$

We assume $\operatorname{mo}_{0}|>| \ell \mid$. The $\phi(\ell, s)$ 's then co:respond to poloidal "sidebands" coupled together by the torcidal effects. Furthermore, we have defined $s=\left(r-r_{0}\right) / \Delta r_{s},\left|r-r_{0}\right| \ll r_{0}, r_{0}$ as the reference mode-rational surface where $m_{0}=n q\left(r_{0}\right), q(r)=$ $\mathrm{rB}_{\mathrm{T}} / \mathrm{RB}_{\ni}$ and $\left(\Delta \mathrm{r}_{\mathrm{s}}\right)^{-1}=\mathrm{m}_{0} \mathrm{q}^{\prime} / \mathrm{q}$. In the present treatment, toroidal coupling enters through the spatial gradients of $B_{O}$. Thus the terms $n_{O} v \cdot v_{E}$ in electron and ion continuity equations are finite and given by

$$
n_{O \sim} \nabla \cdot v_{E} \simeq-i n_{0} w_{d e}\left(\cos \theta+i \frac{r_{0}}{\frac{v_{0}}{\bar{m}_{0}}}(\sin C) \frac{\partial}{\partial r}\right)\left(\frac{e \phi}{T_{e}}\right)
$$

where $\omega_{d e}=\left(C T \mathrm{e}_{0} / \mathrm{m}_{0} \mathrm{Rr}_{0}\right)$. In the electron continuity equation, we also have the following additional term

$$
\nabla \cdot\left(n v_{d e}\right)=i n_{0} \omega_{d e}\left(\cos \theta+i \frac{r_{0}}{m_{0}}\left(\sin \theta \dot{\partial} \frac{\partial}{\partial r}\right)\left(\frac{n}{n_{0}}\right)\right.
$$


One can show using the drift-kinetic approach that these couplings are equivalent to those arising by retention of the magnetic-gradient drifts on the zeroth-order particle trajectories. Following a procedure, similar to that used above in the slab problem one can derive the twodimensional eigen-mode equation given below.

$$
\begin{aligned}
& \left(1-i \frac{w v_{e i}}{k_{\|}^{2} v_{T e}^{2}} \rho^{2}\left[\left[\Delta r_{s}\right)^{-2} \frac{\partial^{2}}{\partial s^{2}}-k_{y o}{ }^{2}\right][\phi(l, s)]\right. \\
& =\left[1-\frac{{ }^{\omega_{*}}}{\omega}-\frac{k_{\|}{ }^{2} c_{s}{ }^{2}}{\omega^{2}}+\frac{{ }^{\omega} \mathrm{de}}{\omega}\left(1-i \frac{{ }^{\omega_{*} \mathrm{e}^{\nu} \mathrm{e}}}{\mathrm{k}_{\|}{ }^{2} \mathrm{v}_{\mathrm{Te}}{ }^{2}}\right) c\right] \phi(\ell, \mathbf{s})
\end{aligned}
$$

where $k_{y o}=m_{0} / r_{0}, k_{\|}=(s-\ell) / q R$, and $c$ is the toroidal coupling operator defined as

$$
\begin{aligned}
& c \phi(l, s)=\frac{1}{2}\{\phi(l+1, s)+\phi(\ell-1, s) \\
& \left.\quad+\frac{q^{\prime} r_{0}}{q} \frac{\partial}{\partial s}[\phi(l+1, s)-\phi(l-1, s)]\right\} .
\end{aligned}
$$

In deriving Eq. (2.12), we have assumed $\left|{ }^{\omega} \mathrm{de} / \omega\right|<1$. 
To make further analytical progress, we reduce the above equation to a one dimensional form by using Taylor's strong coupling approximation [9] $(|\ell|>1)$. We thus seek ballooning-mode-like solutions $[9,16]$ in terms of a single variable $z=\ell-s$ and approximate

$$
\mathrm{c} \phi(l, s) \approx\left[1+\left(\frac{1}{2}-\frac{\mathrm{q}^{\prime} \mathrm{r}_{o}}{\mathrm{q}}\right) \frac{\mathrm{d}^{2}}{\mathrm{dz^{2 }}}\right] \phi(z)
$$

Equation (2.12) now takes the one-dimensional form;

$$
\left[D_{1}\left(1-i D_{3} \frac{z_{R}^{2}}{z^{2}}\right) \frac{d^{2}}{d z^{2}}+D_{2}-\frac{z^{2}}{z_{s}^{2}}-i D_{4} \frac{z_{R}^{2}}{z^{2}}\right] \phi(z)=0,
$$

where

$$
\begin{aligned}
& D_{1}=\frac{\omega}{\omega}\left(\frac{1}{2}-\frac{q^{\prime} r_{0}}{q}\right)-\frac{\rho_{s}^{2}}{\Delta r_{s}^{2}}, \\
& D_{2}=1-\frac{\omega_{* e}}{\omega}+k_{y o}{ }^{2} \rho_{s}{ }^{2}+\frac{\omega_{d e}}{\omega}, \\
& D_{3}=\left[\frac{\omega_{d e^{\omega} *} e}{\omega^{2}}\left[\frac{1}{2}-\frac{q^{\prime} r_{o}}{q}\right)-\frac{\rho_{s}^{2}}{\Delta r_{s}^{2}}\right] / D_{1}, \\
& \mathrm{D}_{4}=\frac{{ }^{\omega} \mathrm{de} \mathrm{w}^{\omega} \mathrm{e}}{\omega^{2}}+\mathrm{k}_{y o}{ }^{2} \rho_{\mathrm{s}}^{2}, \\
& \mathrm{z}_{\mathrm{R}}^{2}=\omega v_{\mathrm{ei}} \mathrm{q}^{2} \mathrm{R}^{2} / \mathrm{v}_{\mathrm{Te}}{ }^{2}
\end{aligned}
$$


and

$$
z_{s}^{2}=\left(q R \omega / C_{s}\right)^{2}
$$

Eluation (2.15) is the relevant eigenmode equation to be solved for investigating the effects of stiong toroidal couplings. For $w_{d e}=0$, it reduies to the equation of the slab case, Eq. (2.4).

\section{MATCHED ASYMPTOTIC TREATMENT}

We first examine the eigenvalue problem for the slab case with $L_{\star}=\infty$, i.e., Eq. (2.4) with $\omega_{*_{e}}$ independent of $x$. After changing variables and rearranging the terms Eq. (2.4) may be rewritten as

$$
\left(\frac{d^{2}}{d \tau^{2}}+\gamma-\frac{\tau^{2}}{4}-\frac{\Lambda}{\tau^{2}+\tau_{R}^{2}}\right) \phi(\tau)=0,
$$

where $\tau=x \exp (i \pi / 4) / \lambda$,

$$
\begin{aligned}
& \lambda^{2}=\rho_{s} x_{s} / 2, \\
& \gamma=i \frac{\lambda^{2}}{\rho_{s}{ }^{2}}\left(1-\frac{\omega_{t_{e}}}{\omega}+k_{y}^{2} \rho_{s}^{2}-\frac{x_{R}^{2}}{x_{s}^{2}}\right) \\
& \tau_{R}^{2}=x_{R}^{2} / \lambda^{2},
\end{aligned}
$$


and

$$
\Lambda=i\left(\frac{x_{R}}{\rho_{s}}\right)^{2}\left(I-\frac{\omega_{*_{e}}}{\omega}-i \frac{x_{R}^{2}}{x_{s}^{2}}\right) .
$$

We solve the eigenvalue problem as posed by Eq. (3.1) by the following matching procedure. In order that the matching procedure be valid, we require

$$
\tau_{R}^{2}=2\left(\frac{v_{e i}}{w_{\star_{e}}}\right)\left(\frac{m_{e}}{m_{i}}\right)\left(\frac{L_{s}}{L_{n}}\right) \ll 1,
$$

In the outer region $\left|\tau^{2}\right| \gg\left|\tau_{R}^{2}\right|$, we have Eq. (3.1) as

$$
\left(\frac{d^{2}}{d \tau^{2}}+\gamma-\frac{\tau^{2}}{4}-\frac{\Lambda}{\tau^{2}}\right\} \Phi_{0}(\tau)=0 \text {. }
$$

The solution which properly decays away for $|x| \rightarrow \infty$ and $\underline{\operatorname{Im} \omega}>0$ is given by

$$
\phi_{0}(\tau)=A_{0}\left(\tau^{2} / 2\right)(2 \alpha+1) / 4 U\left(a, b, \tau^{2} / 2\right) \exp \left[-\tau^{2} / 4\right]
$$

where 


$$
\begin{aligned}
& \alpha=-(1+4 \Lambda)^{1 / 2 / 2,} \\
& b=1+\alpha, \\
& a=(b-\gamma) / 2,
\end{aligned}
$$

and $U$ is the Kumer's Confluent Hypergeometric function. Meanwhile, in the inner region, $|\tau| \sim\left|\tau_{R}\right|$, we have

$$
\left(\frac{d^{2}}{d \tau^{2}}-\frac{\Lambda}{\tau^{2}+\tau_{R}^{2}}\right) \phi_{I}(\tau)=0 \text {, }
$$

and the solution is

$$
\phi_{I}(\tau)=\left(\tau_{I}{ }^{2}-I\right)^{1 / 2}\left[A_{I} P_{V}{ }^{1}(\tau)+{ }^{B}{ }_{I} Q_{V}{ }^{l}\left(\tau_{I}\right)\right],
$$

where

$$
v=-(\alpha+1 / 2),
$$

${ }^{\tau}{ }_{I}=\tau / i \tau_{R}=x \exp (-i \pi / 4) / x_{R}, P_{\nu}{ }^{1}$ and $Q_{\nu}{ }^{1}$ are the Associated Legendre functions. The ratio $B_{I} / A_{I}$ is decided by the parity condition at $x=0$. We have for even- $\phi$ modes, 


$$
\left(B_{I} / A_{I}\right)_{e}=i \frac{2}{\pi} \cos \left(\frac{v \pi}{2}\right) \exp \left(-i \frac{v \pi}{2}\right)
$$

and, for odd- $\phi$ modes,

$$
\left(B_{I} / A_{I}\right)_{0}=-\frac{2}{\pi} \sin \left(\frac{v \pi}{2}\right) \exp \left(-i \frac{v \pi}{2}\right) \text {. }
$$

We note that in deriving Eqs. (3.13) and (3.14), we have only considered the $x \geq 0$ half-space and assumed

$$
\operatorname{Im}\left[x_{R} \exp (i \pi / 4)\right]>0
$$

We now proceed to match $\phi_{0}$ and $\phi_{I}$. First, for $\left|\tau^{2} / 2\right| \ll 1$, we have $\phi_{0}$ as

$$
\vec{b}_{0}^{<}(\tau)=\frac{A_{0}}{\sin \pi b}\left[\frac{\left(\tau^{2} / 2\right)^{-v / 2}}{\Gamma(1+a-b) \Gamma(b)}-\frac{\left(\tau^{2} / 2\right)(1+v) / \overline{2}}{\Gamma(a) \Gamma(2-b)}\right]
$$

where $\Gamma$ denotes the usual Gamma function. Meanwhile, for $\left|\tau_{I}\right|=\left|\tau / \tau_{R}\right| \gg 1, \phi_{I}$ becomes

$$
\begin{aligned}
& \phi_{I}^{>}(\tau)=\frac{{ }^{A_{I}} 2^{\nu}}{\pi / 2}\left[\tau_{I}{ }^{\nu+I} \frac{\Gamma(\nu+1)}{\Gamma(\nu)}+2^{-(1+2 \nu)} \tau_{I}^{-\nu}\right. \\
& \left.\frac{\Gamma(-v-1 / 2)}{\Gamma(-I-v)}\left[1+\pi \frac{{ }^{B} I}{{ }^{A} I} \cot \pi \nu\right)\right] \text {. }
\end{aligned}
$$


Matching $\phi_{0}^{<}$and $\phi_{I}^{>}$then yields the following eigenvalue condition

$\frac{\Gamma(a)}{\Gamma(a r v+1 / 2)}=-\left(\frac{i \tau_{R}}{2^{3 / 2}}\right) 1+2 v \frac{\Gamma(v) \Gamma(-v-1 / 2) \Gamma(1 / 2-v)}{\Gamma(-1-v) \Gamma(v+1 / 2) \Gamma(3 / 2+v)}-\left(1+\pi \frac{B}{A_{I}} \cot \pi v\right)$.

Note the definitions of various symbols here from Eqs.

$(3.2)-(3.5),(3.8)-(3.10)$ and $(3.12)-(3.14)$.

The eigenvalue condition, Eq. (3.18), is in general

difficult to solve analytically for the complex frequency

$\omega$. However, approximate results may be obtained in certain interesting limits:

A. $\quad|\Lambda| \simeq k^{2}\left|x_{R}\right|^{2}<1 / 4$.

In this weakly collisional limit, we have approximately, from Eqs. (3.8) and (3.12)-(3.14),

$\alpha \simeq-(1 / 2)-\Lambda, v \simeq \Lambda,\left\langle B_{I} / A_{I}\right)_{e} \simeq 2 i / \pi,\left(B_{I} / A_{I}\right)_{0}=-v(I-i \nu \pi / 2)$.

For even- $\phi$ modes, Eq. (3.18) thus becomes

$$
\Gamma(a) / \Gamma\left(a+\Lambda+\frac{1}{2}\right) \simeq-2^{3 / 2}\left(\tau_{R} / \pi \Lambda\right) .
$$


Making the further approximation

$\Lambda<T_{R}\left(i . e ., k^{2}\left|x_{R}\right|^{2}<x_{R}\left(\rho_{s} x_{s}\right)^{-1 / 2}<1\right)$, Eq. (3.19) gives

$$
a=-n+(-1)^{n+1} 2^{-3 / 2}(\pi \Lambda) /\left[\tau_{R}\left[\left(-n+\frac{1}{2}\right)\right] .\right.
$$

Concentrating on the lowest $n=0$ even eigenmode and using Eqs. (3.3), (3.9) and (3.10), we obtain the dispersion relation

$$
1-\frac{\omega_{t_{e}}}{\omega}=-\left(i_{\bar{L}_{s}}^{L_{n}}+k_{y}{ }^{2} \rho_{s}{ }^{2}\right)\left[1+\left(\pi \frac{\nu_{e i}}{\omega_{\star_{e}}} \frac{m_{e}}{m_{i}} \frac{L_{s}}{L_{n}}\right)^{1 / 2}\right] .
$$

Equation ( 3.21 ) leads to the surprising conclusion that in a sheared geometry resistivity only contributes to an enhancement of the damping of drift waves. A detailed analysis shows that in this limit, the expected growth terms are proportional to $v_{\text {ei }}$ and always subdominant to the resistivity induced enhanced damping, proportional to $v_{e i}{ }^{1 / 2}$. Thus no unstable eigenmode exists.

As we go to higher collisionalities and higher $k\left|x_{R}\right|$. such that $\left|\tau_{R}\right|<|\Lambda|<1 / 4$, we have from eg. (3.19)

$a \simeq-n-\frac{1}{2}-\Lambda+(-1)^{n+1_{2} 3 / 2 \frac{\tau_{R}}{\pi \Lambda \Gamma(-n-1 / 2)} ; n=0,1 \ldots}$ 
The dispersion relation for the $n=0$ even eigenmode in this limit then becomes

$$
\begin{aligned}
1-\frac{\omega_{*} e}{\omega}= & -\left(k_{y}{ }^{2} \rho_{s}{ }^{2}+i 3 \frac{L_{n}}{L_{s}}\right)-2\left(\frac{\nu_{e i}}{\omega_{*_{e}}} \frac{m_{e}}{m_{i}} \frac{L_{s}}{L_{n}}\right)\left(k_{y}{ }^{2} \rho_{s}{ }^{2}+i \frac{5}{2} \frac{L_{n}}{L_{s}}\right) \\
& -8\left(\frac{L_{n}}{L_{s}}\right)^{2} \pi^{-3 / 2}\left(k_{y}{ }^{2} \rho_{s}{ }^{2}+i 3 \frac{L_{n}}{L_{s}}\right)^{-1}\left(\frac{\nu_{e i}}{\omega_{*_{e}}} \frac{m_{e}}{m_{i}} \frac{L_{s}}{L_{n}}\right)^{-1 / 2} .
\end{aligned}
$$

If we cc pare Eqs. (3.21) and (3.23), we see that the modes are further damped as the resistivity increases. Thus, again th?re is no unstable eigenmode.

Let us now turn to the case of odd- $\phi$ modes; Eq. (3.19) is replaced by

$$
\frac{\Gamma(a)}{\Gamma(a+\Lambda+1 / 2)}=-\frac{\pi \Lambda T R}{2^{1 / 2}}
$$

Noting that $\left|\Lambda \tau_{R}\right| \propto \nu \nu_{\mathrm{ei}}^{3 / 2} \ll 1$, we have, from F, $\mathrm{g}$. (3.?!,

$$
a=-m-\Lambda-1 / 2 ; m=0,1, \ldots
$$

where we have neglected $0\left(v_{\mathrm{e}}^{3 / 2}\right)$ corrections. The dispersion 
relation corresponding to the lowest $(m=0)$ odd eigenmode then is

$$
1-\frac{u_{t_{e}}}{\omega}=-\left(k_{y}{ }^{2} \rho_{s}{ }^{2}+i 3 \frac{L_{n}}{L_{s}}\right)-2\left(\frac{v_{e i}}{\omega_{*}} \frac{m_{e}}{m_{i}} \frac{L_{s}}{L_{n}}\right)\left(k_{y}{ }^{2} \rho_{s}{ }^{2}+i \frac{5}{2} \frac{L_{n}}{L_{s}}\right) .
$$

Here, again, resistivity contributes to the damping of the waves and no unstable eigenmode exists.

$$
\text { B. } \quad|\Lambda| \simeq \mathrm{k}^{2}\left|\mathrm{x}_{\mathrm{R}}\right|^{2}>1 \text { : }
$$

In this strongly collisional limit, we have $\alpha \approx-\Lambda^{1 / 2}$ and $v \simeq \Lambda^{1 / 2}-1 / 2$. Assuming $\operatorname{Im} \Lambda^{1 / 2}<0$, we have with $|\Lambda|>1, \operatorname{Im} v \simeq \operatorname{Im} \Lambda^{1 / 2}<-1$. Equation (3.18), then becomes

$$
\frac{\Gamma(a)}{\Gamma(a+v+1 / 2)}= \pm i\left(\frac{\tau_{R}}{2 / 2}\right)^{1+2 \nu} \frac{\Gamma(v) \Gamma(-v-1 / 2) \Gamma(1 / 2-v)}{\Gamma(-1-v) \Gamma(v+1 / 2) \Gamma(3 / 2+v)} .
$$

Here, + and - correspond to the e ren- and odd- $\phi$ modes, respertively. Applying the Stirling's formula [17] to the Gamma functions, Eq. (3.27) can be further reduced to

$$
\frac{\Gamma(a)}{\Gamma\left(a+\Lambda^{1 / 2}\right)}=\mp i\left(\frac{e \tau}{i 2^{3 / 2} \Lambda^{1 / 2}}\right)^{2 \Lambda^{1 / 2}} \text {. }
$$


If we note that $\left|\tau_{R}^{2} / \Lambda\right| \approx\left(k^{2} x_{s} \rho_{S}\right)^{-1} \ll 1$ and $R e \Lambda^{1 / 2}>1$, Eq. (3.28) gives, for the lowest eigenmode and both parities,

$$
a \simeq-\Lambda^{1 / 2}
$$

The corresponding dispersion relation then is given by

$$
1-\frac{\omega_{\star} e}{\omega}=-k_{y} \rho_{s}\left[k_{y} \rho_{s}+(1+i)\left(2 \frac{\nu_{\text {ei }}}{\omega_{\star_{e}}} \frac{m_{e}}{m_{i}}\right)^{1 / 2}\right] \text {, }
$$

which ciearly shows that the resistive damping effects persist in this limit and no unstable eigenmode exists.

In summary, we have shown that, as long as $\tau_{\mathbf{R}}{ }^{2} \ll 1$, i.e. $\left(v_{e i} m^{L_{s}} s^{\prime \omega_{*}} e^{\left.m_{i} L_{n}\right) \ll 1,}\right.$ electrostatic drift waves in slab geometries and sheared magnetic fields are, in addition to the usual shear-damping effects, further damped by the resistive effects and, thus, no absolutely unstable eigenmode exists. The waves, of course, can still be convectively unstable [5, 14, 15]. Now, a more careful examination of the above analyses reveals that these results are closely related to the fact that drift waves in slab geometries experience shear damping (i.e., the corresponding potential is an anti-well rather than a well). We therefore expect if shear damping can be nullified by, for example, taking $\omega_{\star}$ e to be a localized function of $x$, the stability properties could be qualitatively different. This is what we explore next by solving Eqs. (2.4) and (2.5) with $L_{\star} \neq \infty$. 
A:ter a change of variables and a rearragnert of terms,

Eqs. (2.4) and (2.5) may be rewritten as

$$
\left[\frac{a^{2}}{d \xi^{2}}+\gamma_{0}-\frac{\xi^{2}}{4}-\frac{\Lambda_{0}}{\xi^{2}-i \xi_{R}^{2}}\right] \phi(\xi)=0,
$$

where $;=x / \lambda_{0}, \lambda_{0}^{2}=x_{0} \rho_{s} / 2$,

$$
x_{0}^{-2}=\frac{\omega_{*}}{\omega} \frac{1}{L_{\star}^{2}}-\frac{1}{x_{s}^{2}}
$$

and $\gamma_{0}, \Lambda_{0}, \xi_{R}$ have expressions identical to those lor $\gamma, \Lambda$ and $T_{R}$, Eqs, (3.2) - (3.5), with the substitutions $\lambda_{0}, \omega_{*_{0}}$ and $x_{0}$ for $\lambda$, $\omega_{*_{e}}$ and $x_{s}$. To nullify shear damping, we must obviously require that $\mathrm{R}_{e}\left(\mathrm{x}_{0}^{-2}\right)>0$; i.e., from Eq. (3.32),

$$
L_{*}^{2}<x_{s}^{2}\left(\omega_{* 0} / \omega\right)
$$

If we assume $\left|\xi_{R}\right|^{2} \ll I$, we can use the same matching procedure as the one discussed above for $L_{\star}=\infty$ equation. We can again obtain an eigenvalue condition which has interesting approximate forms in the limits $\left|\Lambda_{0}\right|<1 / 4$ and $\left|\Lambda_{0}\right|>1 / 4$. We now omit the detailed algebra and only present the results of the dispersion relations derived for the lowest eigenmodes. 
For even- $\phi$ modes and with $\left|\Lambda_{\mathrm{O}}\right|<\left|\xi_{\mathrm{R}}\right|<1$, we have

$$
1-\frac{\omega_{\star_{0}}}{\omega} \simeq-\left(k_{y}{ }^{2} \rho_{s}{ }^{2}+\frac{\rho_{s}}{x_{0}}\right)\left[1+\frac{\sqrt{\pi}}{2}(1-i)\left(\frac{v_{\text {ei }}}{w_{\star_{0}}} \frac{m_{e}}{m_{i}} \frac{L_{s}{ }^{2}}{L_{n}} \frac{\rho_{s}}{x_{0}}\right)^{1 / 2}\right] \text {. }
$$

Equation (3.34) shows that when $\mathrm{R}_{\mathrm{e}} \mathrm{x}_{\mathrm{o}}^{-2}>0$, the shear damping is indeed nullified, as expected. Furthermore, resistivity now contributes to instability growth and we have unstable eigenmodes. The growth rate is proportional to $v_{\mathrm{ei}}^{1 / 2}$. As the collisionality is increased, we go to the limit $\left|\xi_{R}\right|<\left|\Lambda_{0}\right|<1$ and the new dispersion relation becomes

$$
\begin{aligned}
& \left.1-\frac{\omega_{*_{0}}}{\omega} \simeq-\left(k_{y}{ }^{2} \rho_{s}^{2}+3 \frac{\rho s}{x_{0}}\right)+2 i \mid k_{y}^{2} \rho_{s}^{2}+\frac{5}{2} \frac{\rho s}{x_{0}}\right)\left(\frac{m_{e}}{m_{i}} \frac{v_{e i}}{w_{*_{0}}} \frac{L_{s} L_{n}^{2}}{L^{2}} \frac{\rho_{s}}{x_{0}}\right) \\
& +\left(\frac{4}{\pi}\right)^{3 / 2}\left[\frac{1+i}{2}\right]\left(\frac{\rho_{s}}{x_{0}}\right]^{2}\left(k_{y^{2}}{ }^{2} \rho_{s}^{2}+3 \frac{\rho_{s}}{x_{0}}\right)^{-1}\left(\frac{m_{e}}{m_{i}} \frac{\nu_{e i}}{\omega_{*_{0}}} \frac{L_{s}}{L_{n}{ }^{2}} \frac{\rho_{s}}{x_{0}}\right)^{-1 / 2}
\end{aligned}
$$

Equation (3.35) shows that as resistivity is increased, the instability growth rate is enhancedand unstable eigenmodes persist as long as $\operatorname{Rex}_{0}^{-2}>0$.

We now investigate the odd- $\phi$ modes. Here the dispersion 
relation is given by

$1-\frac{\omega_{*_{0}}}{u}=-\left(k_{y}{ }^{2} \rho_{s}{ }^{2}+3 \frac{\rho s}{x_{0}}\right)+2 i\left(k_{y}{ }^{2} \rho_{s}{ }^{2}+\frac{5}{2} \frac{\rho s}{x_{o}}\right)\left(\frac{m_{e}}{m_{i}} \frac{\nu_{e i}}{\omega_{*_{0}}} \frac{L_{s}{ }^{2} \rho_{n}{ }^{2}}{x_{o}}\right)$.

where we have neglected corrections of order $v_{e i}{ }^{3 / 2} \cdot$ Equation (3.36) shows that odd- $\phi$ unstable eigenmodes also exist. Finally, we go to the strongly collisional limit, $\left|\Lambda_{0}\right| \simeq k^{2}\left|x_{R}\right|^{2} \gg 1$. Taking $\operatorname{Im\Lambda _{0}} 1 / 2<0$, one can show that the corresponding dispersion relation for both parities takes the form

$$
1-\frac{\omega_{\star_{0}}}{\omega} \simeq-k_{y}{ }^{2} \rho_{s}^{2}+(i-1) k_{y} \rho_{s}\left(\frac{\rho_{s} L_{s}}{x_{0}} \frac{m_{n}}{L_{i}} \frac{v_{e j}}{\omega_{k_{0}}}\right)^{1 / 2} \text {. }
$$

Thus even for strong collisions, resistivity continues to play a destabilizing role and unstable eigenmodes persist.

From the above discussions it is clear that the existence of unstable eigenmodes in the slab approximation with a spatial variation of $\omega_{*_{e}}$ is critically dependent on the condition Eq. (3.33). If Eq. (3.33) is obeyed, shear damping is nullified and resistivity contributes to unstable eigenmodes; otherwise 
only damped sigenmodes exist. Condition (3.33) requires that the density gradient variation saale-length $I_{*}<\rho_{s}\left(I_{s} / L_{n}\right)$, which is rather a sharp variation of the density gradient.

As first pointed out by Taylor [9], shear damping could also be nullified by strong toroidal couplings. We thus expect that resistive drift waves could have unstable eigenmodes in toroidal geometries. This expectation is borne out by the following analysis. The eigenmode equation for the toroidally-coupled mode is given by Eq. (2.15); which, again, can be rewritten in the now familiar form

$$
\left(\frac{\mathrm{d}^{2}}{\mathrm{~d} \tau^{2}}+\gamma_{I}-\frac{\tau^{2}}{4}-\frac{n_{1}}{\tau^{2}-i \tau_{R}^{2}}\right) \phi(\tau)=0
$$

where $\tau=z / \lambda_{1}, \lambda_{1}^{2}=\omega \mathrm{wRD}_{1}{ }^{1 / 2} / 2 \mathrm{C}_{\mathrm{s}}$.

$$
\begin{aligned}
& \gamma_{I}=\left(D_{2}-i D_{3} z_{R}^{2} / z_{s}^{2}\right) \lambda_{1}{ }^{2} / D_{I}, \\
& \Lambda_{I}=i z_{R}{ }^{2}\left(D_{4}-D_{3} D_{2}\right),
\end{aligned}
$$

and $\tau_{R}^{2}=D_{3} z_{R}^{2} / \lambda_{I}^{2}$. The $D^{\prime} s, z_{R}^{2}$ and $z_{s}{ }^{2}$ are defined in Eqs. (2.6) - (2.21). We note that the stability properties as posed by Eq. (3.38) depends critically on the sign of $D_{1}$. 
For $\mathrm{D}_{1}: 0$, Eq. (3.38) is qualititatively the same as that in a slab geometry; i.e., Eq. (2.4). The results are thus similar: i.e., shear damping is operative and the eigenmodes are further damped by the resistivity. We therefore concentrate only on the case with $\mathrm{D}_{1}>0$; i.e., strong toroidal coupling limit where shear damping is nullified. Again, we assume $\left|\tau_{R}^{2}\right| \ll 1$ and solve Eq. (3.38) as done for the slab case. We omit the details and present only the final dispersion relations.

In the weakly collisional limits, $\left|\Lambda_{I}\right|<\left|\tau_{R}\right|<1$, we have for the even- $\phi$ modes

$$
\begin{aligned}
& 1-\frac{\omega^{*} e}{\omega} \simeq-\left(k_{y o}{ }^{2} \rho_{s}^{2}+\varepsilon_{n}\right)+\frac{\varepsilon^{3 / 2}}{2^{1 / 2} q k_{y o} \rho_{s}}+2^{-5 / 4}(i-1) \varepsilon^{1 / 4} \\
& \left(k_{y o}{ }^{2} \rho_{s}^{2}+\varepsilon_{n}\right)\left(\pi q k_{y o} \rho \frac{\nu e_{i}}{w_{* e}} \frac{m_{e}}{m_{i}}\right)^{l / 2}
\end{aligned}
$$

Here, $\varepsilon_{n}{ }^{-1}=\operatorname{Rd\ell n}[\mathrm{N}(r)] / d r$ and we have taken ${ }_{r}{ }_{0} q^{\prime} / q<1 / 2$. Equation $(3.41)$ clearly shows that, with $D_{1} \simeq \varepsilon_{n} / 2>n$, shear damping is indeed nullified by the toroidal cnuplings and unstable eigenmodes do exist. The growth rates scale as $\nu_{e i}^{1 / 2}$. As $\nu_{e i}$ increases such that $\left|\tau_{R}\right|<\left|\Lambda_{l}\right|<1 / 4$, the 
dispersion relation becomes

$$
\begin{aligned}
& 1-\frac{\omega_{* e}}{\omega} \simeq-\left(k_{y \circ}{ }^{2} \rho_{s}^{2}+\varepsilon_{n}\right)+\frac{3 \varepsilon^{3 / 2}}{2^{1 / 2} q k_{y \circ} \rho_{s}}+i\left(\frac{2}{\varepsilon_{n}}\right)^{1 / 2} q k_{y \circ} \rho_{s} \\
& \left(k_{y o}{ }^{2} \rho_{s}^{2}+\epsilon_{n}\right) \frac{v_{e i}}{w_{* e}} \frac{m_{e}}{m_{i}}+\frac{2^{9 / 4}}{\pi / 2} \frac{(i+1)}{\left(k_{y o}{ }^{2} \rho_{s}{ }^{2}+\varepsilon_{n}\right)} \\
& \frac{\varepsilon_{n}^{11 / 4}}{\left(q k_{y o} \rho_{s}\right)^{5 / 2}}\left(\frac{\omega_{*} e}{v_{z i}} \frac{m_{i}}{m_{c}}\right)^{1 / 2} .
\end{aligned}
$$

Thus, the eigenmodes continue to be unstable.

As for the odd- $\phi$ modes, we obtain, in this weakly

collisional limit $\left|\Lambda_{1}\right|<1 / 4$, the following dispersion relation

$$
\begin{aligned}
& 1-\frac{\omega_{*} e}{\omega} \simeq-\left(k_{y o}{ }^{2} \rho_{s}{ }^{2}+\varepsilon_{n}\right)+\frac{3}{21 / 2} \frac{\varepsilon_{n}^{3 / 2}}{q k_{y o}{ }^{\rho} s}+i q\left(\frac{2}{\varepsilon_{n}}\right)^{1 / 2} q k_{y o} \rho_{s} \\
& \left(k_{y o}{ }^{2} \rho_{s}^{2}+\varepsilon_{n}\right) \frac{v_{e i}}{w_{*}} \frac{m_{e}}{m_{i}} \text {. }
\end{aligned}
$$

which indicates that odd- $\phi$ modes also have unstable eigenmodes.

We now discuss the collisional limit, $\left|\Lambda_{1}\right|>1$. In

this limit the dispersion relation for both parities is given by

$1-\frac{\omega_{*} e}{\omega} \simeq-\left(k_{y o}{ }^{2} \rho_{s}{ }^{2}+\varepsilon_{n}\right)+(i+1)\left(k_{y o}{ }^{2} \rho_{s}{ }^{2}+\varepsilon_{n}\right)^{1 / 2}\left(\varepsilon_{n} \frac{\nu_{e i}}{\omega_{* e}} \frac{m_{i} e_{i}}{\pi_{i}}\right)^{1 / 2}$ 
Thus, unstable eigenmodes persist into this strongly collisional regime and the growth rates increases with $\nu_{\text {ei }}$ as $\nu_{e i}^{1 / 2}$.

We thus have shown that, in torodial geometries, toroidalcoupling effects can qualitatively modify the stability propertits of resistive drift-wave eigenmodes. If the toroidal couplungs are strong enough so as to nullify shear damping $\left(D_{1}>0\right)$, then unstable eigenmodes do exist and the growth rates increase witl $v_{e i}$. We note that $D_{1}>0$ requires $r_{0} q^{1} / q<1 / 2$ and $\left(k_{y} \rho_{s} r_{0} q^{\prime / q}\right)^{2}<\varepsilon_{n}\left(1 / 2-r_{0} q^{\prime} / q\right)$. Furthermore, the above results are limited to $\left|\mathrm{T}_{\mathrm{R}}{ }^{2}\right| \ll 1$ or

$$
\frac{v_{e i}}{w_{\star e}} \frac{m_{e}}{m_{i}} \ll \frac{(\varepsilon / 2)^{3 / 2}}{q k{ }_{y o}{ }^{\rho}}
$$

Finally, Taylor's strong-coupling approximations [9] requires $\lambda_{1}^{2}>1$ or $\mathrm{qk}_{\mathrm{yo}} \rho_{\mathrm{s}}>2(2 \varepsilon)^{1 / 2}$.

IV. WKB TREATMENT

Let us consider the eigenvalue problem described by the differential equation

$$
\frac{d^{2} \phi}{d x^{2}}+G(\omega, x) \phi=0,
$$

and the boundary conditions $\phi\left(\phi^{\prime}\right)=0$ at $x=0$ for odd (even) 
modes and well-behavedness as $|x| \rightarrow \infty$ for $\operatorname{Im} \omega>0$. We may use standard phase integral methods (WKB method) o solve this problem if $G^{1} / G^{2} \ll 1$ for the entire domain of $x$. For Eq. (2.4), which is of interest to us, the appropriate conditions are satisfied if $k_{Y}{ }^{2}\left|x_{R}\right|^{2} \gg 1$. Note that this not only overlaps with the $|\Lambda|>1$ approximation of the matched asymptotic treatment in Sec. 3, but indeed extends the analysis to the domain $\left|{ }_{\mathrm{T}}\right|^{2}>1$, where the matched asymptotic treatment breaks down.

The WKB solutions of Eq. (4.I) are

$$
\phi_{ \pm}=G^{-1 / 4} \exp \left[ \pm i \int G^{1 / 2}(\omega, x) d x\right]
$$

A general solution may thus be written as

$$
\phi \approx a_{+} \phi_{+}+a_{-} \phi_{-}
$$

The coefficients $a_{+}$, $a_{-}$are not fixed over the whole complex $z$ plane $(z=x+i y)$ and the $w K B$ method consists in relating, for a given solution of Eq. (4.1), the WKB approximation in one region of the $z$-plane to that in another. A guide to these connection formulae is obtained by mapping out all the zeroes and poles of the function $G(z, \omega)$ in the complex $z$ plane. This is done by drawing the stokes diagram for the function $G(z, w)$, in the standard manner $[18,19]$. Stokes and AntiStokes lines emanating from the zeroes of $G(z, \omega)$ describe the 
global properties of WKB solutions. Along the Anti-stokes lines, the solutions ${ }_{ \pm}$oscillate $i . e .$, have constant amplitude. Having obtained, by examination of the Anti-stokes structure, a pair of zeroes appearing to be associated with a mode, one uses standard connection formulae [18] for qetting the eigenvalue coraition. Notice however that the zesoes of $G$ ani indeed the entire stokes structure itself devends on ui, which has to be finally derived from the eigenvalue conditisn. The problem is therefore often not amenable to analytical treatment. Iterative numerical methods however work very well and a code for carrying out such a complex frequency search, has been developed by white [19]. The final test of the WKB solution is that once the frequency has been determined, the resulting Stokes diagram should be such that a solution can be chosen which satisfies the physical boundary conditions.

For $\mathrm{Eg} .(2.4)$, the function $\mathrm{G}(z, w)$ is given by

$$
G=-\left[k_{y}^{2} \rho_{s}^{2}+\frac{z^{2}}{z{ }^{2}-i x_{R}^{2}}\left(\frac{\omega-\omega_{\star} e}{\omega} \frac{z^{2}}{x_{S}^{2}}\right)\right]
$$

It has two poles and four zeroes given respectively by

$$
z_{\text {pole }}^{2}=\left(\mathrm{ix}_{\mathrm{R}}^{2}\right)
$$


and

$$
z_{\text {zero }}^{2}=\frac{1}{2} x_{s}{ }^{2}\left[k_{y}{ }^{2} \rho_{s}{ }^{2}+\frac{w-w_{\star} e}{w}\right] \pm\left[\frac{1}{4} x_{s} 4\left(k_{y}{ }^{2} \rho_{s}^{2}+\frac{w-\omega_{\star} e}{w}\right)\right.
$$

$$
\left.-i k_{y}^{2} \rho_{s}^{2} x_{R}^{2} x_{s}^{2}\right]^{1 / 2}
$$

The poles and zeroes have obvious symmetries about the origin. Figure I shows a typical stokes diagram for this function. The region $A(B)$ can be shown to include the asymptotic positive (negative) real axis for $\operatorname{Im} \omega>0$. For either Im $\omega>0$ or $\operatorname{Im} \omega<0$ the physical boundary condition demands the choice of a subdominant (decaying) solution in Region A, and the standard connection formula

$$
\int_{z_{1}}^{z_{2}}[G(z, w)]^{1 / 2} d z=\left(n+\frac{1}{2}\right) \pi
$$

produces a subdominant solution extending from $z_{1}$ toward the origin. Thus the solution will approximately vanish at the origin. A numerical integration of $\mathrm{Eq}$. (4.1) would be expected to yield the same eigenvalue as $\mathrm{Eq}$. (4.7), since from Eq. (4.2) the solutions behave as $\phi_{ \pm^{*}} \exp \left( \pm k_{y} \rho_{s} x\right)$ for $\operatorname{small} x$. Any combination of $\phi_{ \pm}$will be completely dominated by $\phi_{+}$ before reaching $z_{1}$ provided only that 
$\left|z_{l}\right|>>\left(k_{y}^{p_{s}}\right)^{-1}$, which is well satisfied.

The insensitivity of the eigenvalue condition, which

guarantees the boundary condition at $x=\infty$, to variations of $\phi$

at $\mathrm{x}=0$ indicates that even and odd modes vill have practically the same eigenvalue. This result is in agreement with the large $\Lambda$ lirnit of matıred asymptotic treatment (Sec. 3) and also numerical calculations (Sec. 6i. Unfortunately, the integration in Eq. (4.7) has to be done numerically so that we do not have a closed analytic form for the eigenvalue condition. Figure 2 presents the results of the WKB analysis for $m_{e^{\prime}} m_{i}=10^{-4}, L_{s} / L_{n}=100$, $\mathrm{k}_{\mathrm{y}}{ }^{2} \rho_{\mathrm{s}}^{2}=.02$ and various values of $\nu_{\mathrm{ei}} / \omega_{*}$. Also presented for comparison are the results obtained from Eq. (3.30) of the matching treatment and those from a direct numerical integration of the differential equation by the shooting method (Sec. 6). Note that there is very good agreement between the various methods.

The above treatment thus permits us to conclude that even for $\left(m_{e}{ }^{\nu} e^{L}{ }_{s} / m_{i} \omega_{*} L_{n}\right)>I$, where the matched asymptotic treatment breaks down, no unstable eigenmodes can be found for resistive drift waves in the slab geometry.

Finally, we wish to present some analytical WKB results in the limit $k_{y}\left|x_{R}\right| \rightarrow \infty$. In this limit $G$ gets very large and the only way to satisfy the eigenvalue conditon Eq. (4.7) is for the two turning points $z_{1}$ and $z_{2}$ to coalesce. From Eq. (4.6), it is readily seen that the condition for the coalescence 
of the two zeroes of $G$ is given by

$$
k_{y}^{2} p_{s}^{2}+\frac{\omega-\omega_{*}}{\omega}= \pm 2 k_{y^{\rho}}{ }_{s}\left(\begin{array}{c}
x_{R}^{2} \\
x_{s}^{2}
\end{array}\right)^{1 / 2}
$$

Substitution of $x_{R}$ and $x_{s}$ gives the eigenvalue conditicn

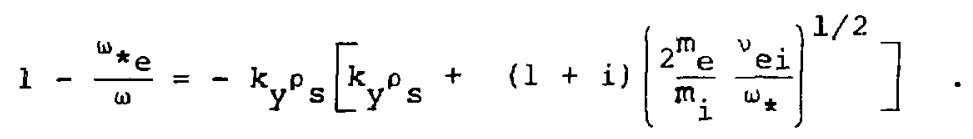

Corrections of order $\left(k_{y}\left|x_{R}\right|\right)^{-1}$ ther split the coalesced turning points apart giving the typical stokes diagram shown in Fig. 1. The sign in front of square-root has been chosen in such a way that the stokes diagram gives the proper boundary condition at $\operatorname{Rez} \rightarrow \infty$. Note that the eigenvalue condition Eq. (4.8) is identical to Eq. (3.30) obtained by the large $k_{y}\left|x_{R}\right|$ Iimit. This suggests that the eigenvalue condition is unaltered by switching the inequality $\left|\tau_{R}^{2}\right|=\left(m_{e} \nu_{e i} L_{s} / m_{i} \omega_{\star} L_{n}\right)<1$, as long as $k_{y}\left|x_{R}\right|>1$ is satisfied. 


\section{PER'TURBATION TREATMENT}

In the previous two sections we have solved the eigenvalue problem associated with Eq. $\{2,4\}$ by non-perturbative matched asymptotic and WKB treatments, respectively. We have found that the two treatments agree in the limit $k_{y}\left|x_{R}\right|>1$. We now demonstrate that the $k_{y}\left|x_{R}\right| \ll<$ I limit of the matched asymptotic treatment can be recovered by a perturbation theoretic analysis. Such an analysis for the collisional problem was first used by Hsu [20]. We shall then use the perturbation - theoretic treatment to analyze a more complicated eigenvalue equation, which arises when electron temperature fluctuations associated with the drift wave as well as finite electron temperature gradients are retained.

We now wish to solve Eq. (2.4) by a perturbation theory in which $\tau_{R}^{2}=\left(m_{e} v_{e i} L_{s} / m_{i} \omega_{*} I_{n}\right) \ll 1$ is treated as the small parameter. We first rewrite Eq. (2.4) in the form Eq. (3.1) with the various quantities defined as before. We note now that for $\tau_{R}=0$, the equation is a parajolic cylinder equation with the usual Hermite function solution

$$
\phi_{n}(\tau)=2^{-n / 2} H_{n}\left(\frac{\tau}{\sqrt{2}}\right) \exp \left(-\frac{\tau^{2}}{4}\right) .
$$

and the eigenvalue given by

$$
\gamma_{n}=n+\frac{1}{2} \quad ; \quad n=0,1,2 \ldots
$$


The ${ }^{2}{ }_{R}^{2}$ terms give a perturbation of the eigenvalue which may be obtained by standard first order perturbation techniques yielding

$$
\delta \gamma_{n}=\left[\int_{-\infty}^{\infty} d \tau \phi_{n}^{2}(\tau)\left(\frac{\Lambda}{\tau^{2}+\tau_{R}^{2}}\right)\right] / \int_{-\infty}^{\infty} d \tau \phi_{n}^{2}(\tau) .
$$

For the lowest $\mathrm{n}=0$ (even- $\phi$ ) mode, we get

$$
\delta \gamma_{O}=i \frac{\lambda^{2}}{\rho_{S}{ }^{2}}\left(\frac{\pi}{2}\right)^{1 / 2}\left(1-\frac{\omega_{\star} e}{\omega}\right) \tau_{R} .
$$

The corresponding dispersion relation is then given by

$$
1-\frac{\omega_{* e}}{\omega}=-\left(k_{y}{ }^{2} \rho_{s}{ }^{2}+\frac{L_{n}}{i_{\frac{L}{L}}}\right)\left[1+\left(\pi \frac{m_{e}}{m_{i}} \frac{\nu_{e i}}{\omega_{\star}} \frac{L_{s}}{L_{n}}\right)^{1 / 2}\right]
$$

Note that this equation is identical to Eq. (3.21) obtained in the matched asymptotic treatment in the $\operatorname{limit} \mathrm{k}_{\mathrm{y}}\left|\mathrm{x}_{\mathrm{R}}\right|<1$. Similar results are obtainable for the odd- $\phi$ modes. We now derive the modification of $\mathrm{Eq}$. (2.4), which includes effects associated with finite electron thermal conductivity along the lines of force and ambient electron temperature gradients. We shall then solve that equation by a perturbation treatment similar to the one given above. The ion dynamics 
and the electron perpendicular (to $\underset{\sim 0}{\mathrm{~B}}$ ) motion is the same as discussed in Sec. 2. The electron parallel equation of motion is modified to [21]

$$
\begin{aligned}
w v_{\| l}=- & k_{\|} e \phi / m_{e}-i v_{e i}\left(v_{l e}-v_{H i}\right) \\
& +k_{\|}\left(T_{e} n_{e} / N_{e}+1.71 t_{e}\right) / m_{e}
\end{aligned}
$$

Here, $t_{e}$ is the perturbed electron temperature. Furthermore, we have the electron equation of energy given by

$$
\frac{3}{2} N_{e}\left(\omega t e-\omega_{k T} e \phi\right)-N_{e} e^{T} e^{k}\left\|^{v}\right\|_{e}=k_{11} q_{e l l},
$$

where

$$
q_{e \|}=0.71 N_{e} \mathrm{~T}^{\mathrm{v}} v_{\| e}-i k_{\| l}{ }^{\mathrm{N}} e^{\mathrm{T}} e^{t} e^{/ m_{e} v_{e k}},
$$

$w_{* T}=-\left(c k_{y} T_{e} / B_{o}\right)\left(d \ell n T e^{/ d x}\right)$ and $v_{e k} \simeq v_{e i} / 1.6$ for singly charged ions. The final (radial) ware equation then can be shown to be

$$
\begin{gathered}
{\left[1+g_{2}(x)\right] \rho_{s}^{2}\left(\frac{d^{2}}{d x^{2}}-k_{y}^{2}\right) \phi(x)=\left\{1-\frac{\omega_{\star} e}{\omega}-1.71 \frac{\omega_{\star T}}{\omega} g_{1}(x)\right.} \\
\left.-\left[1+1.95 g_{1}(x)\right] \frac{x^{2}}{x_{s}}\right\} \phi(x),
\end{gathered}
$$


where

$$
\begin{aligned}
& g_{1}(x)=-i x_{k}{ }^{2} /\left(x^{2}-i x_{k}{ }^{2}\right) \\
& g_{2}(x)=1.95 g_{1}(x)-i x_{R}^{2} / x^{2} .
\end{aligned}
$$

and $\mathrm{x}_{\kappa}{ }^{2}=1.5 w v_{\mathrm{ek}} / \mathrm{k}_{\mathrm{I}}{ }^{2} \mathrm{v}_{\mathrm{Te}}{ }^{2}$

We note that we can recover the resistive wave equation, Eq. (2.4), by setting $x_{K}{ }^{2}=0$ in Eq. (5.8). Again, by changing the variable $x$ and rearranging terms, we can rewrite Eq. (5.8) in the following form

$$
\left[\frac{d}{d \tau^{2}}+\gamma_{k}-\frac{\tau^{2}}{4}-\Sigma_{i} ; \tau_{1} \tau_{\tau}\right]=0
$$

Here, $\tau=x \exp (i \pi / 4) / \lambda, \lambda^{2}=\rho_{s} x_{s} / 2$,

$$
\begin{aligned}
& \gamma_{k}=i\left(\frac{\lambda}{\rho_{s}}\right)^{2}\left(k_{y}^{2} \rho_{s}^{2}+1-\frac{\omega_{\star} e}{\omega}\right), \\
& F(\tau)=i\left(\frac{\lambda}{\rho_{S}}\right)^{2}\left[\left(1-\frac{\omega_{\star} e}{\omega}\right)\left(1.95 \tau_{K}^{2}+\tau_{R}{ }^{2}\right) \tau^{2}+{ }^{2}{ }_{R}{ }^{2} \tau_{\kappa}{ }^{2}\right. \\
& \left.+1.71 \frac{\omega_{\star_{T}}}{\omega} \tau_{K}{ }^{2} \tau^{2}+i\left(\frac{x_{R}}{x_{S}}\right)^{2} \tau^{2}\left(\tau^{2}+\tau_{K}{ }^{2}\right)\right] / g_{3}(\tau),
\end{aligned}
$$




$$
\begin{array}{r}
g_{3}(\tau)=\tau^{4}+\left(2.95 \tau_{K}^{2}+\tau_{R}^{2}\right) \tau^{2}+\tau_{R}^{2} \tau_{K}^{2}, \\
\tau_{R}^{2}=x_{R}^{2} / \lambda^{2}, \text { and } \tau_{K}^{2}=x_{K}^{2} / \lambda^{2} .
\end{array}
$$

Due to the complexities in $F(\tau)$, we have, so far, been unable to solve Eq. (5.11) using the matching procedures developed for the resistive case. Hence, we shall confine our diszussions on Eq. (5.11) to a perturbation analysis, in which both $\tau_{R}$ and $\tau_{K}$ are treated as small parameters. We thus write

$$
\begin{aligned}
& \gamma_{\mathrm{Kn}}=\mathrm{n}+1 / 2+\delta \gamma_{\mathrm{n}} ; \mathrm{n}=0,1,2, \\
& \delta \gamma_{\mathrm{n}}=\left[\int_{-\infty}^{\infty} \phi_{\mathrm{n}}{ }^{2} F(\tau) \mathrm{d \tau}\right] / \int_{-\infty}^{\infty} \phi_{n}{ }^{2} \mathrm{~d} \tau .
\end{aligned}
$$

For the lowest even- $\phi(n=0)$ mode, one obtains

$$
\begin{aligned}
& \delta \gamma_{0}=\left(\frac{\pi}{2}\right)^{1 / 2} \frac{i \lambda^{2}}{\rho_{S}{ }^{2}\left(\tau_{1}+\tau_{2}\right)}\left[\left(1-\frac{\omega_{*} e}{\omega}\right)\left(1.95 \tau_{\kappa}{ }^{2}+\tau_{R}{ }^{2}\right)\right. \\
& \left.+1.71 \frac{\omega_{\star} T}{\omega} \tau_{K}^{2}+\frac{\tau_{R}{ }^{2} \tau_{K}{ }^{2}}{\tau_{1} \tau_{2}}+i \frac{x_{R}{ }^{2}}{x_{s}{ }^{2}}\left(\frac{2}{\pi}\right)^{1 / 2}\left(\tau_{1}+\tau_{2}\right)\right],
\end{aligned}
$$


where

$$
\tau_{1,2}^{2}=\left\{2.95 \tau_{K}^{2}+\tau_{R}^{2} \pm\left[\left(2.95 \tau_{K}^{2}+\tau_{R}^{2}\right)^{2}-4 \tau_{R}^{2} \tau_{K}^{2}\right]^{1 / 2}\right\}^{\prime 2}
$$

The corresponding dispersin relation that is given

$$
\begin{aligned}
& 1-\frac{\omega_{*} e}{\omega} \simeq-\left(k_{y}{ }^{2} \rho_{s}{ }^{2}+i \frac{L_{n}}{L_{s}}\right)-\left(\frac{\pi}{2}\right)^{1 / 2}\left[( k _ { y } { } ^ { 2 } \rho _ { s } ^ { 2 } + i _ { \frac { L _ { s } } { L _ { s } } } ) ^ { 2 } \left(1.95 \tau_{k}{ }^{2}\right.\right.
\end{aligned}
$$

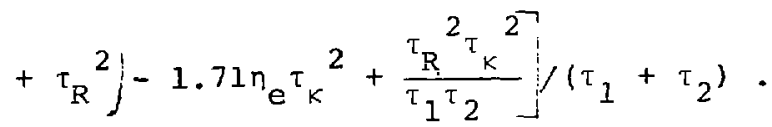

Here, $\eta_{e}=\omega_{*_{T}} / \omega_{*_{e}}=d \ell n T_{e}(x) / d \ell n_{e}(x)$.

Noting that $\tau_{1}{ }^{2}, \tau_{2}{ }^{2}, \tau_{R}{ }^{2}, \tau_{\kappa}{ }^{2} \propto \nu_{\in i}$, Eq. (5.19) indicates that dissipative effects further enhance the shear damping rates by factors proportional to $v_{e i}^{1 / 2}$. It is also interesting to note that electron temperature gradient (the $\mathrm{n}_{\mathrm{e}}$ term) only modifies the real frequencies and has no effects on stability of the eigenmode.

As to the $n=1$ eigenmodes; i.e., the lowest odd- $\phi$ mode, we have,

$$
\delta \gamma_{1} \simeq i\left(\frac{\lambda}{\Gamma_{s}}\right)^{2}\left[\left(1-\frac{\omega_{\star} e}{\omega}\right)\left(1.95 \tau_{k}{ }^{2}+\tau_{R}{ }^{2}\right)+i\left(\frac{x_{R}}{x_{s}}\right)^{2}+1.71-\frac{\omega_{* T}}{\omega} \tau_{\kappa}{ }^{2}\right],
$$


and the corresponding dispersion relation is

$$
\begin{aligned}
1-\frac{w_{\star} e}{\omega} \simeq & -\left(k_{y}{ }^{2} \rho_{s}{ }^{2}+i 3 \frac{L_{n}}{L_{s}}\right)\left(1+1.95 \tau_{k}{ }^{2}\right)+1.71 \eta_{e}{ }^{1}{ }^{2} \\
& -\left(k{ }_{y}{ }^{2} \rho_{s}{ }^{2}+i \frac{5}{2} \frac{L_{n}}{L_{s}}\right) \tau_{R}{ }_{R} .
\end{aligned}
$$

Equation (5.21) indicates that dissipative effects again only enhance the shear damping rates by factors proportional to $v_{e i}$ and that finite $\eta_{e}$ only modifies the real frequencies. Thus, using a perturbation analysis, we have demonstrated that finite electron thermal conductivity along the lines of force and amiient temperature gradients do not alter the stability properties of dissipstive drift waves in a slak-cieometry sheared magnetic field. Specifically, we find that electron thermal conductivity further damps the waves in a fashion similar to the resistive effects. Meanwhile, electron temperature gradients are found to only modify the real frequencies and hence, have no effects on the stability.

VI. NUMERICAL RESU:TS AND DISCUSSIONS

We have now demonstrated by several analytical treatments that dissipative drift waves in a plasma slab with sheared magnetic fields do not form unstable eigenmodes in any domain of collj.sionality. Instability is recovered only if the conventional shear damping effects are nullified eithır by localjzing the density gradient or by including strong 
toroidal coupling effects. Since the various analytic treatments have restricted dow ins of validity in parameter space, we have verified and extended many of the results by a direct numerical integration of the differential equation, Eq. (2.4), using the so-called "shooting method". The numerical integration of the equation utilizes a Numerov finite difference scheme. Making a guess about the eigenvalue, the WKB solution for $x$ is utilized for initializing the integration scheme. The equation is integrated to the origin where the derivative $d \phi / d x$ (if we are looking for even modes) or $\phi$ (for odd modes) is ascertained. The deviation of either $\mathrm{d} \phi / \mathrm{dx}$ or $\phi$ (as the case may be) fron zero is essentially used in a Muellex's root-finding package for predicting a new eigenvalue. This is then used as a new guess and the iteration is continued until convergence i: obtained. In the present problem, in view of the fact that the effective potential at large $x$ is an anti well, and that for damped modes the solution finds to diverge in $x$, it turns out to be more convenient to integrate along the $-45^{\circ}$ line in the complex plane. The transformation is chosen such that the outward propagating wave along the real axis goes into the subdominant solution along the $-45^{\circ}$ line. Figure 2 presents a plot of the numerically obtained rate $\gamma / \omega_{*}$ vs. the collision frequency, $v_{e i} / \omega_{*}$ for $k_{y}^{2} \rho_{s}^{2}=0.2$ $\mathrm{m}_{\mathrm{e}} / \mathrm{m}_{\mathrm{i}}=10^{-5}, \mathrm{~L}_{\mathrm{s}} / \mathrm{L}_{\mathrm{n}}=125$. Also plotted in Fig. 2 are 
the results of various analytic treatments. Te see that the agreement is very good. We note, as mentioned previously, that the perturbation theory gives reasonable results when $\left.\left|\Lambda_{i}=\mathrm{k}_{\mathrm{y}}{ }^{2}\right| \mathrm{x}_{\mathrm{R}}\right|^{2}$ is small and the WKB theory is reasonable when $|A|>1$. The physical reason for this is clear from Eq.(3) and (4). In these figures we have plotted the real and jmaginary parts of the eigenfunction along $T$ (viz the $-45^{\circ}$ degree line in the complex $x$ plane, along which the solutions decay) for various yalues of $|\Lambda|$. It is noted that for $\left|\Lambda_{i}\right|$ small, the eigenfunctions are very close to the unperturbec form viz hermite Eunctions. On the other hand for i 1 l large, the eigenfunctions are strongly distorted and have no yesemblance to the unperturbed Hermite functions. They, however, tend to get localized in small regions of $\tau$-axis and hence make the WKB approximation good. It is also interesting to note that in this limit, the eigenfunction almost vanishes near the origin; chus the boundary condition at the origin does not piay a very important role and the odd- and even- parity solutions satisfy the same eigenvalue conditon [of Eq. (3.30) anr] (4.7)]. Very recently we have been able to find a quadratic form whirh demonstrates that Eq. (2.4) has no unstable eigenmodes. The procedure followed below was inspired by a trick used by Antonsen [22] for the corresponding collisionless problem. The existence of this quadratic form then puts the above analyses on a firm footing. Let us transform the independent variable 
$x$ to $s$ defined by

$$
x^{2}=-i x_{R}^{2} s^{2}
$$

The outgoing solution at large $x$ vizexp $\left(-i x^{2} / x_{s}\right)$ goes to a decaying solution $\left.\exp \left(-i\left(m_{e} / m_{i}\right)\left(\nu_{e i} / \omega_{*}\right)\left(L_{s} / L_{n}\right)\right] s^{2}\right)$ along the $s$ direction. Equation (2.4) now takes the form

$$
\frac{d^{2} \phi}{d s^{2}}+\left\{i k^{2} x_{R}^{2}+\frac{s^{2}}{s^{2}+1}\left[\frac{i\left(w-w_{*}\right)}{\omega} \frac{x_{R}^{2}}{\rho_{s}^{2}}-\left(\frac{m_{i}}{m_{i}} \frac{w_{e i}}{w_{*}} \frac{L_{s}}{L_{n}}\right)^{2} s^{2}\right]\right\}=0
$$

Multiplying (6.2) by $\phi^{*}$, integrating all over $s$, using $\omega=\Omega+i \gamma$ and separating the real part we find

$$
\begin{aligned}
& -\int\left|\frac{\partial \phi}{\partial s}\right|^{2} d s+\int\left[-\gamma k^{2} \rho_{s}^{2}\left(\frac{m_{e}}{m_{i}} \frac{\nu_{e}}{\omega_{*}} \frac{L_{L}}{L_{n}{ }^{2}}\right]-\frac{s^{2}}{s^{2}+1}\right. \\
& \left.\left(\gamma \frac{m_{e}}{m_{i}} \frac{\nu_{e i}}{\omega_{t_{e}}} \frac{L_{s}{ }^{2}}{L_{n}{ }^{2}}+\frac{m_{e}^{2}}{m_{i}^{2}} \frac{\nu_{e i}{ }^{2}}{\omega_{*_{e}}{ }^{2}} \frac{L_{s}{ }^{2}}{L_{n}{ }^{2}} s^{2}\right)\right]|\phi|^{2} d s=0 \text {. }
\end{aligned}
$$

For $\gamma>0$, all the above terms are individually negative definite and so their sum cannot go to zero. The conclusion then is that there can be no growing modes for $\mathrm{Eq} .(2.4)$.

We now present a possible physical mechanism explaining the results. An examination of the local dispersion relation for dissipative drift waves indicates that $\omega \simeq \omega_{*}$ only for values of $k_{\|}$such that $\left(k^{2} v_{e T}^{2} / v_{e i} \omega_{*}\right)>\left(k_{y}^{2} \rho_{s}^{2}\right)$. This is the range $k_{\|}$in which the electron motion along the lines of force gives predominantly the usual Boltzmann response $n / n_{0} \simeq e \phi / T$, which is essential for drift waves. 


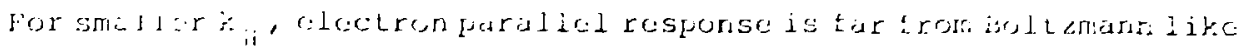

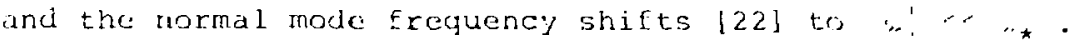
In a shored marnetic Eield geometry, $k_{\|}$takes all values Erom zero up. Thus approximately, within the resistive layer

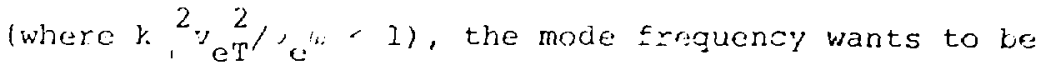
$\therefore . .$. and outside, it wants to be ." '" . Concentrating on modes with in " " * then produces solutions which localize themselves away from the mode-rational surface and tho resistive layer (this is emphasized in the plots in Fig. 4, which are valid for large $\left.k_{y}\left|x_{R}\right|\right)$. Thus, considerable changes ir : and : take place in the transition from the inside to the outside of the resistive layer. This then has severe consequences on shear darping (which is governed by the usual paraboli: cylinder equation but with effectively a new bounciary condition on the inside) and enhances it. This enhancement always overpowers the subdominant growth terms, so we have only damped eigenmodes. However, if one gets rid of the shear danping by some physical effucts which localize the mode ene:gy, then the instability can be recovered sinse the above mechanism does not operate.

The above discussion brings out the sensitivity of the eigenmode analysis to processes occuring within a small resistive layer near the mode-rational surface. It is tempting to speculate that physical processes which might mix the electron fluid over this region (e.g., small magnetic islands, convective cells etc.) will provide the electrons with an effective $k_{\|}$ 
in the inner region which is not too small; this might, in turn, drastically alter the conclusions of the eigermoar analysis. An investigation of these effects is in proguess. Finally, a few words about experiments. Experimejts on drift waves in the collisional regime have been done in stellarators [24] and multjpoles [25]. We have recently shown [26] that the Kharkov stellarator experiments [24] can be explained in terms of a density gradient localized mode. Similar conclusions about the FM-l experiment camnot be firm because no direct measurement of strong density gradients was made (although indirect evidence [27] exists from collisionless instability experiments). Finally, we must always leave open the possibility that the observed fluctuations were associated with convectively amplified wave-packets.

\section{ACKNOWLEDGMENTS}

We wish to thank $\mathrm{H}$. Selberg for help in developing the numerical code for the shooting method, S. Mahajan for discussions at initial stages of the work, A. Hassam and R. Kulsrud for pointing out the importance of some terms in the toroidal coupling treatment, and P. H. Rutherford and M. Kruskal for useful discussions on the WKB analysis.

*This work was supported by the United States Department of Energy Contract No. EY-76-C-02-3073 and the United States Air Force Office, Scientific Research Contract No. F 44620-75-c-0037. 


\section{REFERENCES}

[1] Kadomtsev, B. B., Plasma Turbulence, Academic Press, $(1965), 88$.

[2] Rutherford, P. H. and Frieman, E. A., Phys, Fluids 19, (1967) 1007 .

[3] Kadomtsev, B. B. and Pogutse, O. P., Reviews of Plasma Piys., Consultants Bureau, $\underline{5}$, (1970) 249.

[4] Pearlstein, L. D. and Eerk, H. L. Phys. Rev. Letter's, 23. (1969) 220 .

[5] Coppi, B., Phys. Letters 14, iis65) 172.

[6] Rukhadze, A. A. and Silin, V. P., Sov. Phys. Usp. 11 (1969), 659 [USP. Fix. Nauk 96, (1968) 877.

[7] Krall, N. A. and Rosenbluth, M. N., Phys. Fluids 8, (1.965) 1488 .

[8] Moiseev, S. S. and Sagdeev, R. Z., Sov. Phys. Tech. Phys. 9, (1964) 196 [Ah. Tekh, Fiz. 34, (1964) 248].

[9] Taylor, J. B. Proc. of IAEA Conf. on Plasma Physics and Controlled Fusion Reasearch, Berchetsgarden, 2 (1967) 323.

[10] Ross, D. W. and Mahajan, S. M., Phys, Rev. Letters 40, (1978) 324 .

[11] Tsang, K. T., Catto, P. J., Whitson, J. C. and Smith, J., Phys. Rev. Letters 40 , (1978) 327.

[12] Chen, Liu, Guzdar, P. N., White, R. B., Kaw, P. K. and Oberman, $\dot{\mathrm{C}} .$, Princeton University Plasma Physics Lab. Report No, PPPL-1446, (1978).

[13] Rosenbluth, M. N, and Catto, P. J., Nucl. Fusion 15, (1975) 573; Gladd, N. T. and Horton, W., Phys. J'luids 16, (1973) 879 . 
[14] Cappi, B., Laval, G. Peliat, R. and Rosenbluth, M. N., Nuc1. Fusion $\underline{6},(1966) 261$.

[15] Kent, A. and Taylor, J. B., Phys. Fluids 12, (1969) 209.

[16] Dobrott, D., et al., Phys Rev. Letters 30, (1977) 943.

[17] Abramowitz, M. and Stegon, I. A., Handbook of Mathematical Functions, NBS Appl. Math. Series 55 (196/) 257.

[18] Heading, J., An Introduction to Phase Integral Methocs (John Wiley and Sons, NY) 1962.

[19] White, F. B., Princeton University Plasma Physics Lab. Report PPPL-1482 (1978).

[20] Hsu, J., PhD. Thesis, Princeton University (1977), unpublished.

[21] Tsai, S. T., Perkins, F. W. and Stix, T. H., Phys. Fluids 13, (1970) 2108 .

[22] Antonsen, T. Jr., Proceedings of the Annual Controlled Fusio: _onference, Gatlinburg, Tennessee, April, 1978.

[23] Mahajan, S. M., Guzdar, P. N., Kaw, P, K. and obecman, C. Princeton University Plasma Phys. Lab, Rep., PPPL-1351 (1977).

[24j Vojtsenya, V. S., Voloshko, A. Yu., Zalkind, V. M.. Solodovchenko, S. I., Tarasenko, V. P., Stan, A. Z., Nuc1. Fusion 17, (1977) 651 .

[25] Okabayashi, M. and Arunasalam: V., Nucl. Fusion 17, (1977) 497.

[26] Guzdar, P. N., Chen, Liu, Kaw, P. K. and oberman, C., Phys. Rev. Letters 40, (1978) 1566.

[27] Sauthoff, N., Ph.D. Thesis, Princeton University (1975) Unpublished. 
Fig. 1. Anti-Stokes plot for the function $G(x, w)$ defined in Eq. (44) .

Fig. 2. Comparison of $-\gamma / \omega_{*}$ vs $\nu_{e i} / \omega_{*}$ curve obtained by numerical shooting mehtod and analytic (WKB and matched asymptotic) theories.

Fig. 3. Real and Imaginary parts of the eigenfunction vs. distance along the $-45^{\circ}$ line (i.e., along a line in the complex $x$ plane where the solution decays at large $\mathrm{x}$ ) for $|\Lambda| \ll 1$.

Fig. 4. Same as in Fig. 3 but for $|\Lambda|>>1$. 
$-47-$

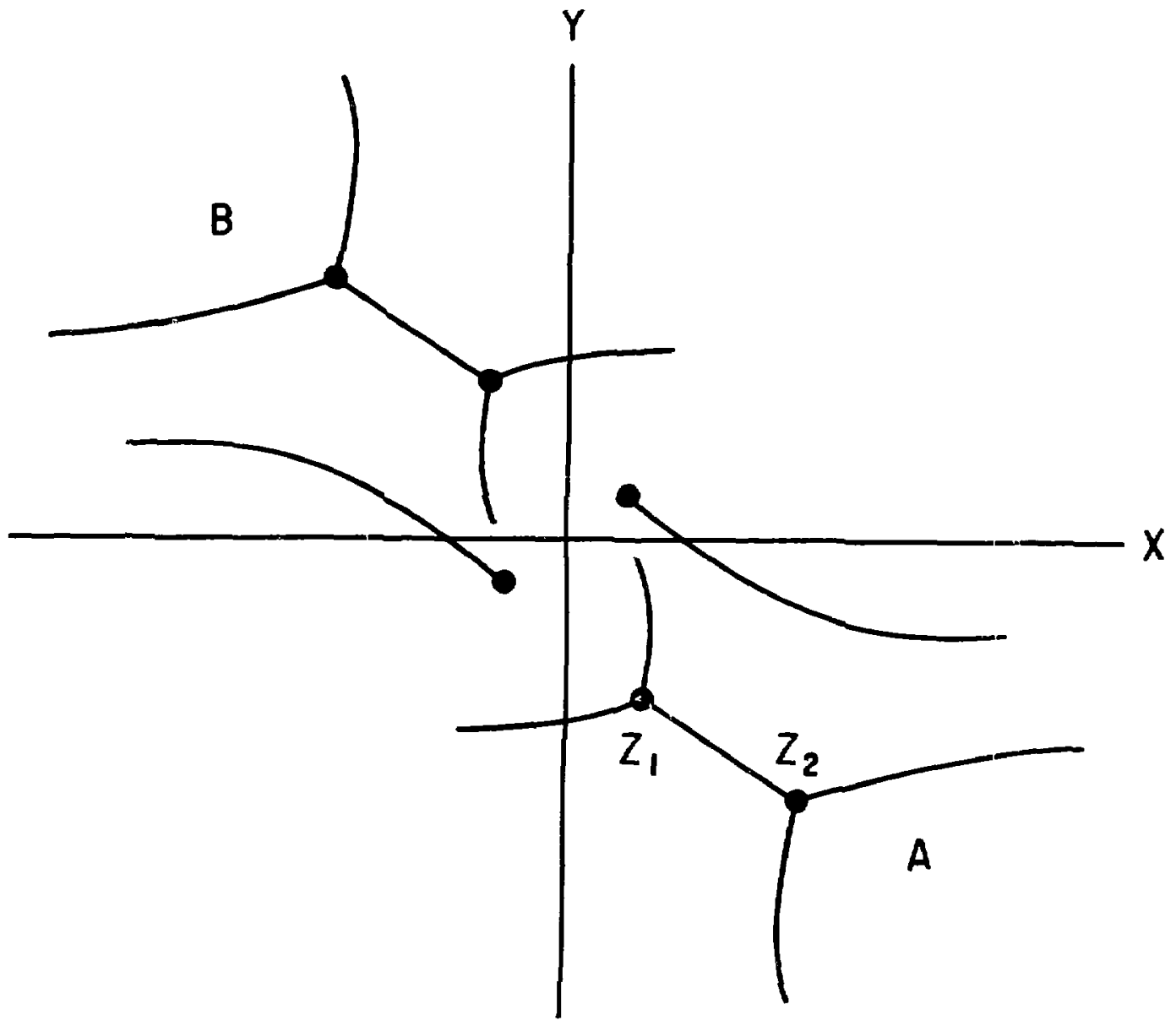

Fig. 1. 782128 


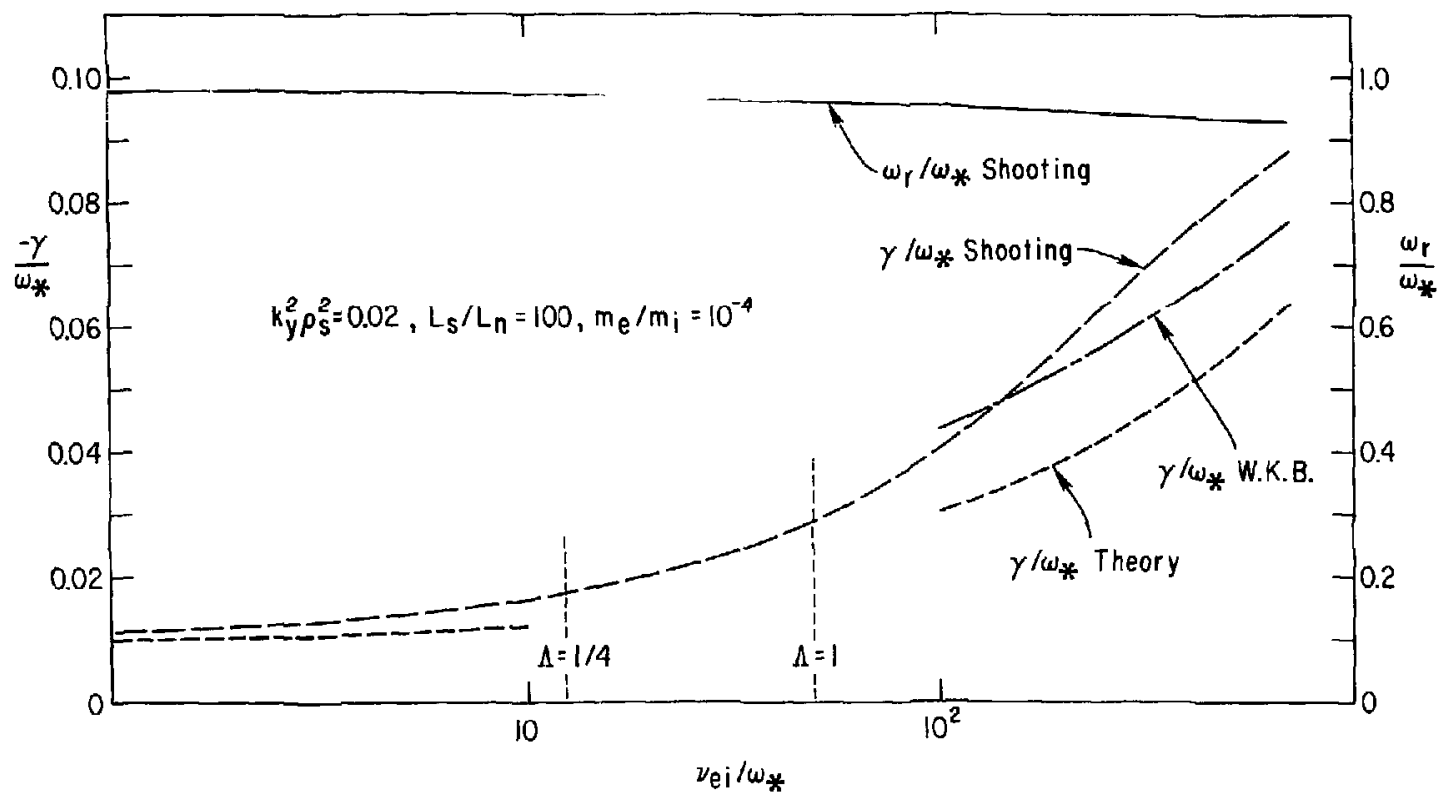




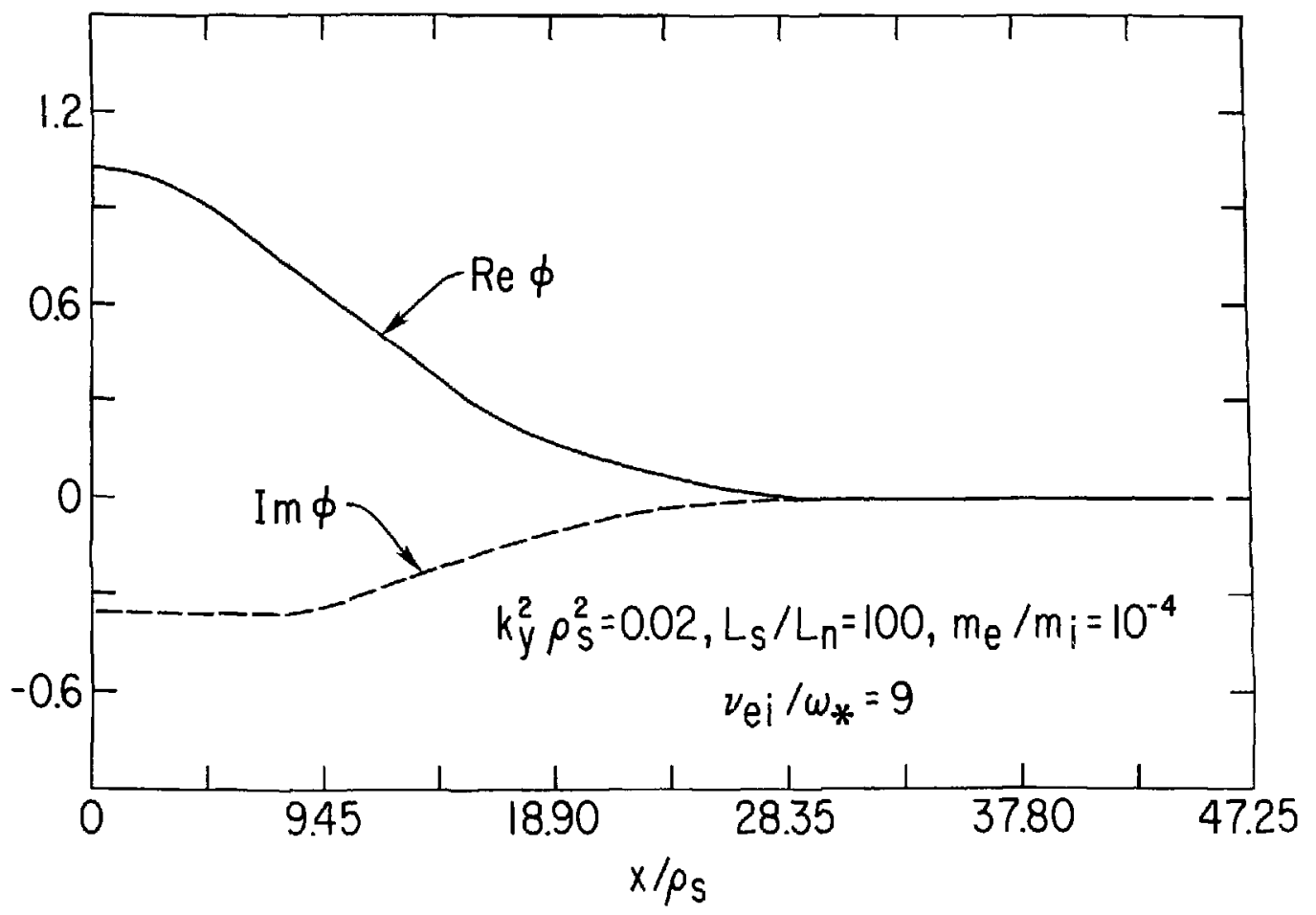

Fig. 3. 782129 


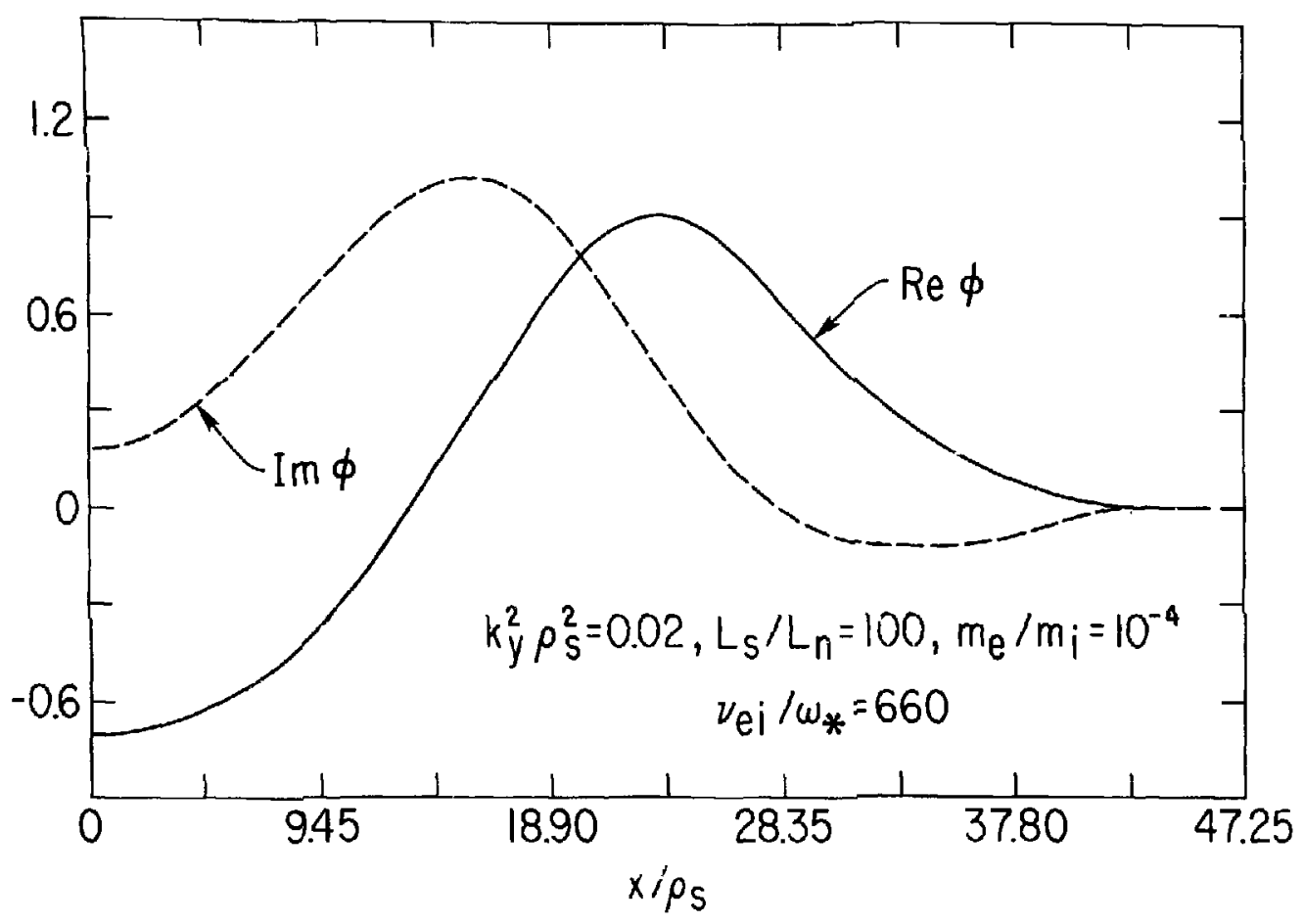

